\title{
Severe disturbance overflows the stabilizing buffer of variable biotic interactions
}

\author{
Koya Hashimoto ${ }^{1,2, * \dagger}$, Daisuke Hayasaka ${ }^{1, \dagger}$, Yuji Eguchi ${ }^{3}$, Yugo $\mathrm{Seko}^{3}, \mathrm{Ji} \mathrm{Cai}^{4}$, \\ Koichi Goka ${ }^{2}$ and Taku Kadoya ${ }^{2, \dagger}$ \\ *For correspondence (atrophaneura4@gmail.com (K. Hashimoto)) \\ †These authors contributed equally to this work.
}

\begin{abstract}
Recent studies have uncovered that biotic interaction strength varies over time in real ecosystems intrinsically and/or responding to anthropogenic disturbances. Little is known, however, about whether such interaction variability strengthens or weakens community resistance against disturbances. Here, we examine how the change in interaction strength after pesticide application mediates disturbance impacts on a freshwater community using outdoor mesocosms. We show that the change in interaction strength buffered the disturbance impact but amplified it once the disturbance severity exceeded a certain threshold. Importantly, we also show that interactions fluctuating more temporally under no disturbances were more changeable in response to pesticide applications. Our findings suggest that a severe disturbance may have a surprise impact on a biological community amplified by their own interaction variability, but the possibility still remains that we can predict the consequences of the disturbance by measuring the interaction variability before the disturbance occurs.
\end{abstract}

\section{Keywords}

anthropogenic disturbances; community resistance; community stability; empirical dynamic modelling; herbicides; insecticides; interaction strength; mesocosm experiment; pesticides 


\section{Author affiliation}

1: Faculty of Agriculture, Kindai University, Nakamachi 3327-204, Nara, 631-8505, Japan.

2: National Institute for Environmental Studies (NIES), Onogawa 16-2, Tsukuba, Ibaraki 305-8506, Japan.

3: Graduate School of Agriculture, Kindai University, Nakamachi 3327-204, Nara, 6318505, Japan.

4: Center for Ecological Research, Kyoto University, Hirano 2-509-3, Otsu, Shiga 5202113, Japan.

\section{Introduction}

Predicting the magnitude to which anthropogenic disturbances would hurt ecosystems is essential for successful conservation and ecosystem management. In real ecosystems, all members of a biological community are linked with each other by biotic interactions such as predation, competition and mutualism, and these interactions often cause nonlinear, unpredictable responses of biological communities to a disturbance regardless of whether the disturbance is natural or anthropogenic (Rohr et al. 2006; Suttle et al. 2007; Doak et al. 2008). Indeed, ecologists have considered that community responses to disturbances highly depend on the strength of biotic interactions (McCann et al. 1998; Ives \& Carpenter 2007; Vellend 2016). For example, weak interactions can improve community stability (measured by resistance/resilience to a disturbance) by muting potentially unstable, strongly interacting community members (McCann et al. 1998; McCann 2000; Kadoya \& McCann 2015). Notably, many earlier studies postulated a 'static view' of interaction networks, focusing on long-term averaged properties of interactions responsible for community stability (May 1972; Dunne 2006; Allesina \& Tang 2012). More recently, however, researchers have begun to recognize 
that interaction strength can be highly variable due to trait plasticity and/or density changes in response to intrinsic and extrinsic factors, including anthropogenic disturbances (Tylianakis et al. 2008; Ohgushi et al. 2012; Ushio et al. 2018; Bartley et al. 2019). This view of variable interactions urges us to reconsider our understanding of how interaction strength affects community responses to anthropogenic disturbances.

Although there have been several attempts to incorporate interaction variability into community stability theory (e.g., de Ruiter et al. 2005; Neutel et al. 2007; Rooney et al. 2008; McMeans et al. 2015), previous studies have provided contrasting predictions regarding whether interaction variability improves or impairs community resistance/resilience to external disturbances. For example, flexible foraging by predators can make food webs more resistant to disturbances (Kondoh 2003; Rooney et al. 2006, 2008; Loeuille 2010), partly because flexible foragers can change predation rates in response to changes in their prey density such that the collapse of prey populations is avoided. Empirical evidence of stabilization by interaction variability also exists; in a predator-prey relationship between whelks and barnacles, for example, the reduced predation of barnacles by whelks resulted in the quick recovery of barnacle populations after artificial disturbances that generated bare rocks (Navarrete \& Berlow 2006). These studies suggest that changes in interaction strength may generate 'buffering effects' that absorb the impacts of external disturbances. However, it has been anticipated that changes in interaction strength due to external disturbances can 'amplify' rather than buffer the harmful impacts of the disturbances (Rooney et al. 2006; Calizza et al. 2017). For example, chemical pollution, including agrochemicals, can disturb the behaviour of individuals, such as foraging and defence, thereby altering trophic interactions and thus their vital rates (e.g., survival and development), in addition to the effects of direct chemical toxicity (Relyea \& Hoverman 2006). Additionally, it has been reported that habitat degradation due to urbanization decreased food availability for marine arthropods such as shrimp, leading to stronger competition 
and lower stability (Calizza et al. 2017). Thus, our knowledge about whether interaction variability tends to buffer or amplify the impacts of disturbances is still limited.

Here, we explored the role of interaction variability in determining community responses to anthropogenic disturbances. We combined a manipulative mesocosm experiment and nonlinear time-series analysis to track variability in interaction strength. As a model system, we chose pesticides and freshwater communities in paddy ponds, the representative agricultural habitats in East Asia. We conducted three-year outdoor mesocosm experiments where the applications of an insecticide (fipronil) and an herbicide (pentoxazone) were fully crossed with two replicates each. During the experimental period (approx. 140 days) in each year, we monitored the density of ten community members, including phyto- and zooplankton, plants and macroinvertebrates (for the biological information of community members, see APPENDIX 3), every two weeks. Based on the fortnight-interval data, we quantified the strength of interactions among these community members and their variability by empirical dynamic modelling (EDM), a recently developed analytical framework for nonlinear time-series data (Chang et al. 2017). EDM is a powerful approach that can track time-varying interaction strength by using only time series of the density of each community member (Deyle et al. 2016; Ushio et al. 2018).

Specifically, to assess whether variable interactions buffered or amplified the pesticide impacts on the density of the community members, we examined the relationships among the following: (1) 'interaction strength (IS) responses' to the pesticides measured as changes in the IS of each interaction link due to pesticide disturbances; (2) mean and temporal variability of each IS without pesticides (we refer to the latter as 'IS intrinsic variability' hereafter); and (3) changes in the density of the community members included in the corresponding interaction pair due to pesticides. We first showed that IS responses to pesticides indeed buffered the disturbance impacts on community members' density but shifted to an amplification effect once the 
disturbance became more severe. We then showed that intrinsically variable interactions (i.e., variable interactions even under no disturbance conditions) were more likely to exhibit larger IS responses to pesticide impacts, thereby indirectly regulating community resistance against pesticide disturbances. Finally, we discuss how our results contribute to a more holistic view of interaction variability-community stability relationships. We suggest that measuring IS intrinsic variability before disturbances occur might be useful to predict consequences of future disturbances for communities and ecosystems.

\section{Results}

Pesticide impacts on the density of each paddy community member

For the majority of community members, insecticide applications had stronger impacts on their density than did herbicide applications (Fig. 1a, Table S1). Insecticide treatment dramatically decreased the density of phytophilous and benthic predatory insects (i.e., Odonata larvae) [C (control) vs. I (insecticide alone)], which was consistent with previous studies (Hayasaka et al. 2012a; Hashimoto et al. 2019). Additionally, although not statistically significant, the insecticide treatment decreased detritivorous insects and increased neustonic predatory insects and molluscs. The herbicide treatment significantly decreased only the density of macrophytes [C vs. H (herbicide alone)]. When we applied both the insecticide and the herbicide, phytoplankton, macrophytes, herbivores, phytophilous predators, and benthic predators significantly decreased in density, and molluscs significantly increased in density [C vs. I+H (mixture of insecticide and herbicide)]. Absolute changes in density of each community member due to the pesticide treatments were largest for $\mathrm{I}+\mathrm{H}$ treatments, and then $\mathrm{I}$ and $\mathrm{H}$ in this order (Fig. 1b), suggesting that the pesticide impacts on the density of paddy community members were larger for this insecticide than for this herbicide. The observed pesticide impacts would be due to direct toxicity, indirect toxicity via biotic 
interactions, and the mixture of these factors.

\section{Quantifying interaction variability}

Our EDM analyses successfully reconstructed the interaction network among paddy community members in each treatment, thereby revealing the responses of IS to pesticide applications (Fig. 2, Table S2, Fig. S2). Figs. 2a and b show the interaction network in the controls. Note that these arrows represent the net effects of interactions (i.e., the sum of direct and indirect effects) rather than the sole direct effects. Most of the interactions were biologically interpretable; for instance, phytoplankton positively affected rotifers, and rotifers negatively affected phytoplankton but positively affected crustacean zooplankton, suggesting prey-predator interactions (Fig. 2a, APPENDIX 4). As several previous studies indicated (Wootton \& Emmerson 2005), the distribution of the mean IS under no disturbances was skewed towards the weaker IS (Fig. 2b), and this tendency did not change for the pesticide-applied treatments (Fig. S2). For every treatment, the numbers of negative and positive interactions were similar (Fig. S2). The differences in IS among the treatments were mostly subtle, but there were several interactions where even the sign (positive or negative) changed when the pesticides were applied (Fig. S2). The absolute differences in IS between the pesticide treatments and the controls (IS responses to the pesticides) were largest when both insecticide and herbicide were applied (i.e., $\mathrm{I}+\mathrm{H}$ treatments) and smallest when the herbicide was applied alone (i.e., H treatment) (Fig. 2c).

IS changed not only in response to pesticide applications but also varied over time within each year even without pesticides. Notably, the magnitude of IS fluctuations differed greatly by different interaction pairs (Figs. 3a, b). For example, the strength of the interaction effects on herbivorous insects showed only subtle fluctuations, but those on molluscs greatly fluctuated over time (Fig. 3b). We refer to these fluctuations in the controls as IS intrinsic variability, as this temporal variability can be regarded as an IS 
characteristic intrinsic to the community in the absence of disturbances. In other words, there were interactions that were intrinsically more variable and less so in the communities in the experimental paddies.

\section{Mediation of pesticide impacts by interaction variability}

Intriguingly, we found nonlinear, concave relationships between IS responses and density changes in the corresponding interaction recipient (i.e., receivers of interaction effects) due to the pesticides (Figs. 4a-c, Table S4). Specifically, when the IS responses were within the range from small to intermediate (up to approx. $1.0 \times 10^{-3}-3.0 \times 10^{-3}$ ), the absolute changes in recipient density due to the pesticide decreased with increasing corresponding IS responses. This finding suggests that IS responses buffered pesticide impacts on each community member. However, when the IS responses exceeded this range, density changes increased with increasing IS responses, suggesting that IS responses amplified pesticide impacts. Although the shapes of the relationships were qualitatively the same (i.e., concave shape) among the three pesticide-applied treatments, ranges showing positive relationships between IS responses and density changes (i.e., amplifying) were wider for the I and $\mathrm{I}+\mathrm{H}$ treatments than for the $\mathrm{H}$ treatment. In addition, the changes in donor (i.e., initiators of interaction effects) density due to the pesticides were not significantly associated with those in recipient density for any of the treatments (Table S4).

Finally, based on structural equation modelling, we found that the IS intrinsic variability was more responsible than the mean IS for determining the IS responses to pesticides and thus indirectly mediated density changes in community members due to pesticides. Specifically, the IS intrinsic variability was strongly and positively associated with IS responses to pesticide applications (Figs. 4d-f, Table S5), while the mean absolute IS in the controls did not have significant effects on the IS responses to pesticides (Fig. 5). In addition, the direct effects of the mean and variability of IS in the 
controls on density changes due to pesticides were not significant $(\mathrm{I}$ and $\mathrm{I}+\mathrm{H})$ or were significant but weak (H) (Fig. 5). Therefore, indirect pathways of IS intrinsic variability $\rightarrow$ IS responses to pesticides $\rightarrow$ absolute changes in recipient density due to pesticides were the dominant linkages in determining the resistance of community members to pesticides (Fig. 5). These results indicate that IS intrinsic variability was the primal predictor of IS responses to pesticide applications and therefore of the resistance of the community against pesticide disturbance.

\section{Discussion}

Our findings have shown that the responses of IS to pesticides certainly mediated the resistance of each community member against pesticide applications, but this mediation showed unimodal shapes, not monotonically buffering or amplifying pesticide impacts. We suggest that this nonlinear relationship between IS response and community resistance to disturbance may underly well-known, nonlinear ecosystem responses to disturbances (often brought by human activities), where a drastic shift in community or ecosystem states occurs when disturbance severity exceeds a threshold or 'tipping point' (Scheffer et al. 2001; Polasky et al. 2011). Specifically, the switch of interaction variability effects on community resistance from buffering to amplification at a certain disturbance threshold may be one mechanism underlying the ecosystem's tipping point behaviour along with disturbance severity. Note that ranges showing positive relationships between IS responses and density changes (i.e., amplifying) were wider when the insecticide (fipronil) was applied than when the herbicide (pentoxazone) was applied. Previous studies reported that fipronil exerts a severe adverse impact on paddy animal communities, especially on the density of Odonata larvae, and drastically alters community structure (Hayasaka et al. 2012a, b; Kasai et al. 2016; Hashimoto et al. 2019). Therefore, the result corresponds well with the existing knowledge that the application of this insecticide causes larger changes in the density of and IS among 
community members in our mesocosms, which mainly consisted of invertebrate animals, than did the herbicide (Figs. 1b, 2c). Given this severe impact of this insecticide, our results suggest that IS responses to disturbances are more likely to amplify the disturbance impacts rather than buffer them when a disturbance is more severe, thereby impairing the resistance of the whole community.

Several processes can be responsible for IS responses to external disturbances, including adaptive trait plasticity, direct disturbance impacts such as sublethal effects of pesticides, and density changes of members of the communities. Therefore, it is not feasible to specify the mechanism underlying the observed IS responses to pesticides in this experiment. Nevertheless, flexible foraging by invertebrate predators and consumers may be one of the possible factors generating the observed IS responses to pesticides. This view is supported by the fact that our additional analysis revealed that IS intrinsic variability was the largest when recipients were predators and the lowest when producers (Table S6, Fig. S4) and that there were tight positive correlations between IS responses to the pesticides and IS intrinsic variability (Figs. 4d-f). These results suggest that flexible foraging by micro- and macroinvertebrates (including apex predators such as dragonflies and consumer organisms such as snails) may have made IS more intrinsically variable, thereby generating larger IS responses. Note that previous studies have often suggested that adaptive, flexible foraging has a stabilizing force (Kondoh 2003; Rooney et al. 2006, 2008; Loeuille 2010; but see Abrams 2010), while there is an existing study showing that severe habitat degradation in marine ecosystems made adaptive foragers consume wider diets, leading to unstable food webs (Calizza et al. 2017). Such mixed evidence for the consequences of flexible foraging may be well interpreted in our comprehensive view, showing that the effects of IS responses on the pesticide resistance of communities are nonmonotonic. Collectively, adaptive responses of individuals could sometimes lead to unstable communities to which these individuals belong, and our finding supports this view. Given that ongoing global environmental 
change is causing interaction rewiring due to the adaptive responses of animals (Bartley et al. 2019), our study warns that such interaction rewiring may have the potential to amplify rather than buffer the impacts of environmental changes when these impacts are too severe.

In this study, the mean IS in the pesticide-free condition did not show significant direct or indirect effects on the resistance of each community member. However, this result does not necessarily mean that ISs themselves were not responsible for community resistance to pesticides. Although the direct path from mean IS to IS intrinsic variability was not significant in the SEM analysis, the relationship between them was negative and triangular, where the upper bound of IS variability was clearly decreased (i.e., constrained) with increasing mean IS (Fig. S5). Thus, the mean IS may have determined the upper bound of IS intrinsic variability rather than the variability on the average, thereby indirectly regulating community resistance to pesticides. Berlow (1999) also observed similar relationships between the mean and variability of IS, where some weak interactions on the average were extremely variable among spatial replicates but strong interactions were less variable; the author argued that the variability of weak interactions should play an important role in community and ecosystem organization. In this context, our study highlights a novel mechanism of community stability in which some weak interactions are allowed to vary in response to external disturbances, thereby mediating community resistance to disturbances. Considering the interaction variability-mediated regulation of community stability is a promising avenue for future research aiming to achieve a more holistic view of the stability-interaction relationship.

Finally, our study has implications for effective ecosystem management. The tight association between IS responses to pesticides, which directly regulated community resistance to pesticides, and IS intrinsic variability (Figs. 4d-f) suggests that IS intrinsic variability may be an effective proxy of IS responses to disturbances. Since 
IS variability without disturbances can obviously be measured before disturbances will take place, measuring IS intrinsic variability may be useful for predicting responses of the focal community to anthropogenic disturbances. Specifically, our results indicate that a community with a higher IS intrinsic variability would be more resistant to a moderate disturbance but less so or even vulnerable to a severe disturbance. Measuring IS intrinsic variability is not a trivial task but can be achieved by recently developed techniques such as the EDM framework. Combining this new technique with knowledge about the roles of interaction variability in determining community stability, we would be able to predict which communities are more sensitive to anthropogenic disturbances, and our study provides the first stepping stone using the EDM framework effectively for such purposes. This approach would serve to improve novel ecological risk assessments, contributing to effective ecosystem management to preventing the system from crossing the tipping point in the future.

\section{Methods}

\section{Experimental design}

This study is a continuation of our previous study (Hashimoto et al. 2019), which examined the single and combined effects of an insecticide and an herbicide on freshwater communities in paddy mesocosms. We chose fipronil and pentoxazone as test chemicals because both are commonly used in Japanese crop management, and their physicochemical properties are as typical as the properties of pesticides used in Japanese paddies (Japan Plant Protection Association 2011). Fipronil acts upon the central nervous systems of animals by inhibiting GABA receptors, which are dominant in the nervous systems of arthropods (Gant et al. 1998). It is relatively stable in water and easily adsorbed to sediment. In sediment, it persists for a quite long time and degrades to some toxic metabolites (Simon-Delso et al. 2015). Pentoxazone is an inhibitor of chlorophyll biosynthesis (Hirai et al. 2001). It is quickly degraded in water 
by hydrolysis but is readily adsorbed to sediment, in which its toxicity to plants persists for a relatively long time (Japan Plant Protection Association 2011; Nagai et al. 2011). Importantly, the direct toxicity of fipronil and pentoxazone is selective; fipronil is highly toxic to arthropods but moderately toxic to other animals and plants, while pentoxazone suppresses many vascular plants, but its toxicity to animals is low.

Experimental procedures were mostly described in Hashimoto et al. (2019). In March 2017, we buried eight independent, fibre-reinforced plastic (FRP) tanks (280 cm length $\times 120 \mathrm{~cm}$ width $\times 40 \mathrm{~cm}$ depth, RK 3014, KAISUIMAREN Co., Ltd., Toyama Prefecture, Japan) in the ground on the campus facilities of Kindai University (Nara prefecture, Japan). Then, we spread sediments of uncontaminated areas near the study site on the bottom of each tank (approximately $30 \mathrm{~cm}$ depth). Each tank was randomly assigned to one of the four treatments, i.e., C: controls, I: insecticide alone, H: herbicide alone, and $\mathrm{I}+\mathrm{H}$ : mixture of insecticide and herbicide.

During 2017-2019, we repeated the following procedure. In mid-April, the mesocosms were flooded with dechlorinated water to a depth of approximately $5 \mathrm{~cm}$. From the end of May to early June, we transplanted insecticide-treated and control rice seedlings (Hino-hikari variety) with an array of $4 \times 10$ at 25 -cm intervals. We applied an insecticide (fipronil) and an herbicide (pentoxazone) in the same way as recommended for commercial rice fields. We treated nursery boxes of rice seedlings with Prince ${ }^{\circledR}(1 \%$ granular fipronil, HOKKO Chemical Industry, Inc., Tokyo, Japan) at a rate of $50 \mathrm{~g} / \mathrm{box}$ $24 \mathrm{~h}$ prior to transplanting. Immediately after transplanting rice seedlings, we applied Sainyoshi flowable ${ }^{\circledR}(8.6 \%$ pentoxazone, KAKEN Pharmaceutical Co., Ltd., Tokyo, Japan) at a rate of $1.7 \mathrm{~mL} / \operatorname{tank}$ (i.e., $500 \mathrm{~mL} / 10$-a) to the mesocosms. We confirmed that these pesticide applications succeeded by monitoring the temporal dynamics of the water and sediment concentrations of the insecticide and herbicide (Fig. S1). The experiment was terminated in mid-October (i.e., approx. 140 days). During the experiment, we monitored the density of each community member every two weeks 
throughout the approx. 140-day experimental period until harvest in each experimental year. Detailed monitoring methods are described in APPENDIX 1. As such, we acquired 8 tanks $\times 3$ years $=24$ fragments of time series, each of which had 10 consecutive timepoints, for each of the 10 community members. Time series of the density data were standardized to zero means and unit variances before the analyses (Chang et al. 2017).

\section{Statistical analysis}

Pesticide impacts on density

All statistical analyses were performed using the statistical environment ' $R$ ' version 3.6.3 (R Core Team 2020). We examined the effects of the pesticide applications on each member's density by using linear mixed models (LMMs) with the package 'glmmTMB' version 1.0.2.1 (Brooks et al. 2017). LMMs contained standardized density as a response variable and treatment and census week (categorical) as explanatory variables. The random part of the models was as follows: we specified tank identity and year as random intercepts and assumed an AR-1 temporal correlation structure within a given tank within a year. Statistical significance of the fixed terms was tested by type III likelihood ratio tests, followed by Dunnett-type post hoc pairwise comparisons (two-sided) with the package 'emmeans' version 1.5.2.1 (Lenth 2020). To examine the differences in treatment impacts on the density of each community member, we compared the absolute density changes in each community member due to the three pesticide treatments by LMMs. Changes in the density of each community member were calculated by subtracting the mean density in treatments $\mathrm{I}, \mathrm{H}$, or $\mathrm{I}+\mathrm{H}$ from that of the controls for each year. Thus, the community members observed in this study were replicates for this analysis. The LMMs contained year as a random intercept, and the statistical significance of treatment comparisons was tested by type III likelihood ratio tests followed by Tukey-type post hoc pairwise comparisons (two-sided). 


\section{Empirical dynamic modelling}

To examine how pesticide applications altered the biotic interactions within the communities in the experimental paddies, we performed EDM analyses. Using a time series of ten members of paddy communities, we first detected dynamic causality by performing convergent cross-mapping (CCM) (Sugihara et al. 2012) to determine interacting pairs of community members and their interaction directions. Then, we performed a multivariate S-map (Deyle et al. 2016) to track time-varying interaction strength among interacting pairs determined by CCM. Detailed methods are described in APPENDIX 2. All EDM analyses were performed by using the package 'rEDM' ver.

\subsection{5 (Ye et al. 2020).}

\section{Detecting causalities by CCM}

To determine if one community member had a dynamic causality on another, we performed CCM analysis (Sugihara et al. 2012). CCM is a recently developed analysis used to test causalities between two variables that potentially interact with each other. In short, CCM examines the correspondence among reconstructed attractor manifolds to test causalities among variables. We tested the causalities of all pairs of 10 community members (eukaryotic phytoplankton, rotifers, crustacean zooplankton, macrophytes, detritivorous insects, herbivorous insects, phytophilous predatory insects, benthic predatory insects, neustonic predatory insects and molluscs).

Tracking IS variability by multivariate S-map

To estimate the IS among community members in the experimental paddies at each time point, we performed a multivariate S-map using causally interacting pairs determined by CCM. The multivariate S-map is a locally weighted sequential linear regression at each location of an attractor manifold reconstructed by multivariate embedding. Deyle et al. (2016) claimed that regression coefficients of the S-map ('S-map coefficients') at 
each time point can be interpreted as 'sequential Jacobian elements' measuring timevarying, population-level interaction strength (i.e., the slope of the numerical response of a recipient community member to a donor community member at each time). These correspond to the net effects of one unit of an increase in a donor on one time-step population growth in a recipient. For an S-map model to predict community member A, estimated S-map coefficients of community member B are regarded as the time-varying interaction strength of community member B on A. Note that S-map analyses were performed for reconstructed manifolds embedded by using the whole data of eight paddies and three years as a composite. Thus, we obtained interaction strength not only in real time but also for every paddy (and thus every treatment) and every year (Fig. S3). To examine the differences in IS response to the pesticide among the treatments, we compared the absolute IS changes in each interaction due to the three pesticide treatments by LMMs. For this analysis, the interaction effects observed in this study were replicates. Changes in IS were calculated by subtracting the mean S-map coefficient in treatments $\mathrm{I}, \mathrm{H}$, or $\mathrm{I}+\mathrm{H}$ from that of the controls for each year. LMMs contained year as a random intercept, and the statistical significance of treatment comparisons was tested by type III likelihood ratio tests followed by Tukey-type post hoc pairwise comparisons (two-sided).

Mediation of pesticide impacts by IS variability

To determine whether the IS responses to pesticides were associated with the pesticide resistance of community members, we performed LMM analyses. We constructed LMMs examining whether changes in recipient density due to pesticides were associated with corresponding IS responses to pesticides. The LMMs were constructed for each treatment comparison (i.e., $\mathrm{I}-\mathrm{C}, \mathrm{H}-\mathrm{C}$, and $\mathrm{I}+\mathrm{H}-\mathrm{C}$ ). The models took the changes in recipient density as a response variable and used the corresponding IS responses and donor density changes as explanatory variables. To account for nonlinear 
relationships, we included a quadratic term of IS responses in the models. The identity of interacting pairs of community members and year were specified as random intercepts. Statistical results are shown in Table S4.

Next, we explored what factors determined the IS responses to pesticide treatments. We constructed LMMs containing the IS responses as a response variable for each treatment comparison. Their explanatory variables were the mean and SD of the S-map coefficients in the controls (the latter is 'IS intrinsic variability'), the mean and SD of the donor and recipient density in the controls, the function (producers, prey, or predators) of donors and recipients, and the numbers of causal links of donors and recipients. The identity of interacting pairs of community members and year were specified as random intercepts. Then, we performed backward stepwise model selection based on Akaike information criterion (AIC). Statistical results are shown in Table S5.

Finally, to explore direct and indirect relationships among the parameters of IS in the controls (i.e., no pesticide disturbance), IS responses and absolute changes in recipient density due to the pesticide treatments, we performed structural equation modelling using the package 'piecewiseSEM' version 2.1.0 (Lefcheck 2016). To account for nonlinear relationships, we preliminarily included a quadratic term for every explanatory variable. When quadratic terms were not significant or when they were significant but the corresponding relationships showed monotonic increases or decreases, the quadratic terms were removed. Correlations were assumed between linear and quadratic terms. The identity of interacting pairs of community members and year were specified as random intercepts. To facilitate interpretation, we scaled and centred all variables before introducing quadratic terms, which enabled us to compare path coefficients among different paths (Schielzeth 2010). Statistical results are shown in Table S7. For all the analyses in this section, the interaction effects observed in this study were replicates. 


\section{Acknowledgements}

Dr. Masayuki Ushio (Kyoto University) assisted greatly with helping our EDM analyses. We are particularly grateful to the members of the laboratory for the conservation ecology of Kindai University for helping our field experiment and survey. We also thank Dr. Robin J. Smith (Lake Biwa Museum) for identifying zooplankton crustaceans, especially ostracods. The authors wish to thank Dr. Tadao Kitagawa for valuable technical advice. The members of the Biodiversity Assessment and Projection Section and the Climate Change Impacts Assessment Research Section of NIES greatly helped us by joining discussions. The present study was supported by the Environment Research and Technology Development Fund (ERTDF) FY2017 (4-1701) of the Ministry of the Environment, Japan, and the Japan Society for the Promotion of Science (JSPS) KAKENHI (grant numbers 20K15640 and 21J01194 to K. Hashimoto and 21K18318 to D. Hayasaka and T. Kadoya).

\section{References}

Abrams, P.A. (2010). Implications of flexible foraging for interspecific interactions: lessons from simple models. Funct. Ecol., 24, 7-17.

Allesina, S. \& Tang, S. (2012). Stability criteria for complex ecosystems. Nature, 483, 205-208.

Bartley, T.J., McCann, K.S., Bieg, C., Cazelles, K., Granados, M., Guzzo, M.M., et al. (2019). Food web rewiring in a changing world. Nat. Ecol. Evol., 3, 345-354.

Berlow, E.L. (1999). Strong effects of weak interactions in ecological communities. Nature, 398, 330-334.

Breheny, P. \& Burchett, W. (2017). Visualization of regression models using visreg. $R$ $J ., 9,56$. 
Brooks, M.E., Kristensen, K., van Benthem, K.J., Magnusson, A., Berg, C.W., Nielsen, A., et al. (2017). glmmTMB balances speed and flexibility among packages for zero-inflated generalized linear mixed modeling. $R J ., 9,378-400$.

Calizza, E., Costantini, M.L., Careddu, G. \& Rossi, L. (2017). Effect of habitat degradation on competition, carrying capacity, and species assemblage stability. Ecol. Evol., 7, 5784-5796.

Chang, C.-W., Ushio, M. \& Hsieh, C. (2017). Empirical dynamic modeling for beginners. Ecol. Res., 32, 785-796.

Deyle, E.R., May, R.M., Munch, S.B. \& Sugihara, G. (2016). Tracking and forecasting ecosystem interactions in real time. Proc. R. Soc. B Biol. Sci., 283, 20152258.

Doak, D.F., Estes, J.A., Halpern, B.S., Jacob, U., Lindberg, D.R., Lovvorn, J., et al. (2008). Understanding and predicting ecological dynamics: Are major surprises inevitable? Ecology, 89, 952-961.

Dunne, J.A. (2006). The network structure of food webs. In: Ecological Networks:

Linking Structure to Dynamics in Food Webs (eds. Pascual, M. \& Dunne, J.A.). Oxford University Press, New York, pp. 27-86.

Gant, D.B., Chalmers, A.E., Wolff, M.A., Hoffman, H.B. \& Bushey, D.F. (1998). Fipronil: action at the GABA receptor. In: Pesticides and the Future (eds. Kuhr, R.J. \& Motoyama, N.). IOS Press, Amsterdam, pp. 147-156.

Hashimoto, K., Eguchi, Y., Oishi, H., Tazunoki, Y., Tokuda, M., Sánchez-Bayo, F., et al. (2019). Effects of a herbicide on paddy predatory insects depend on their microhabitat use and an insecticide application. Ecol. Appl., 29, e01945.

Hayasaka, D., Korenaga, T., Sánchez-Bayo, F. \& Goka, K. (2012a). Differences in ecological impacts of systemic insecticides with different physicochemical properties on biocenosis of experimental paddy fields. Ecotoxicology, 21, 191-201. Hayasaka, D., Korenaga, T., Suzuki, K., Saito, F., Sánchez-Bayo, F. \& Goka, K. (2012b). Cumulative ecological impacts of two successive annual treatments of 
imidacloprid and fipronil on aquatic communities of paddy mesocosms.

Ecotoxicol. Environ. Saf., 80, 355-362.

Hirai, K., Yano, T., Ugai, S., Yoshimura, T. \& Hori, M. (2001). Development of a herbicide, pentoxazone. J. Pestic. Sci., 26, 194-202. (in Japanese)

Ives, A.R. \& Carpenter, S.R. (2007). Stability and diversity of ecosystems. Science, $317,58-62$.

Japan Plant Protection Association (JPPA). (2011). Pesticide handbook 2011. Japan Plant Protection Association, Tokyo, Japan. (in Japanese)

Kadoya, T. \& McCann, K.S. (2015). Weak interactions and instability cascades. Sci. Rep., 5, 12652.

Kasai, A., Hayashi, T.I., Ohnishi, H., Suzuki, K., Hayasaka, D. \& Goka, K. (2016).

Fipronil application on rice paddy fields reduces densities of common skimmer and scarlet skimmer. Sci. Rep., 6, 23055.

Kondoh, M. (2003). Foraging adaptation and the relationship between food-web complexity and stability. Science, 299, 1388-1391.

Lefcheck, J.S. (2016). PIECEWISE SEM : Piecewise structural equation modelling in R for ecology, evolution, and systematics. Methods Ecol. Evol., 7, 573-579.

Lenth, R. V. (2020). emmeans: Estimated Marginal Means, aka Least-Squares Means. Loeuille, N. (2010). Consequences of adaptive foraging in diverse communities. Funct. Ecol., 24, 18-27.

May, R.M. (1972). Will a large complex system be stable? Nature, 238, 413-414.

McCann, K., Hastings, A. \& Huxel, G.R. (1998). Weak trophic interactions and the balance of nature. Nature, 395, 794-798.

McCann, K.S. (2000). The diversity-stability debate. Nature, 405, 228-233.

McMeans, B.C., McCann, K.S., Humphries, M., Rooney, N. \& Fisk, A.T. (2015). Food web structure in temporally-forced ecosystems. Trends Ecol. Evol., 30, 662-672.

Nagai, T., Ishihara, S., Yokoyama, A. \& Iwafune, T. (2011). Effects of four rice paddy 
herbicides on algal cell viability and the relationship with population recovery. Environ. Toxicol. Chem., 30, 1898-1905.

Navarrete, S.A. \& Berlow, E.L. (2006). Variable interaction strengths stabilize marine community pattern. Ecol. Lett., 9, 526-536.

Neutel, A.M., Heesterbeek, J.A.P., Van De Koppel, J., Hoenderboom, G., Vos, A., Kaldeway, C., et al. (2007). Reconciling complexity with stability in naturally assembling food webs. Nature, 449, 599-602.

Ohgushi, T., Schmitz, O. \& Holt, R.D. (Eds.). (2012). Trait-Mediated Indirect Interactions: Ecological and Evolutionary Perspectives. Cambridge University Press, Cambridge, U.K.

Polasky, S., Carpenter, S.R., Folke, C. \& Keeler, B. (2011). Decision-making under great uncertainty: Environmental management in an era of global change. Trends Ecol. Evol., 26, 398-404.

R Core Team. (2020). R: A language and environment for statistical computing. $R$ Foundation for Statistical Computing.

Relyea, R.A. \& Hoverman, J. (2006). Assessing the ecology in ecotoxicology: A review and synthesis in freshwater systems. Ecol. Lett., 9, 1157-1171.

Rohr, J.R., Kerby, J.L. \& Sih, A. (2006). Community ecology as a framework for predicting contaminant effects. Trends Ecol. Evol., 21, 606-613.

Rooney, N., McCann, K., Gellner, G. \& Moore, J.C. (2006). Structural asymmetry and the stability of diverse food webs. Nature, 442, 265-269.

Rooney, N., McCann, K.S. \& Moore, J.C. (2008). A landscape theory for food web architecture. Ecol. Lett., 11, 867-881.

de Ruiter, P.C., Wolters, V., Moore, J.C. \& Winemiller, K.O. (2005). Food web ecology: playing Jenga and beyond. Science, 309, 68-71.

Scheffer, M., Carpenter, S., Foley, J.A., Folke, C. \& Walker, B. (2001). Catastrophic shifts in ecosystems. Nature, 413, 591-596. 
Schielzeth, H. (2010). Simple means to improve the interpretability of regression coefficients. Methods Ecol. Evol., 1, 103-113.

Simon-Delso, N., Amaral-Rogers, V., Belzunces, L.P., Bonmatin, J.M., Chagnon, M., Downs, C., et al. (2015). Systemic insecticides (Neonicotinoids and fipronil): Trends, uses, mode of action and metabolites. Environ. Sci. Pollut. Res., 22, 5-34. Sugihara, G., May, R., Ye, H., Hsieh, C., Deyle, E., Fogarty, M., et al. (2012).

Detecting causality in complex ecosystems. Science, 338, 496-500.

Suttle, K.B., Thomsen, M.A. \& Power, M.E. (2007). Species interactions reverse grassland responses to changing climate. Science, 315, 640-642.

Tylianakis, J.M., Didham, R.K., Bascompte, J. \& Wardle, D.A. (2008). Global change and species interactions in terrestrial ecosystems. Ecol. Lett., 11, 1351-1363.

Ushio, M., Hsieh, C., Masuda, R., Deyle, E.R., Ye, H., Chang, C.-W., et al. (2018). Fluctuating interaction network and time-varying stability of a natural fish community. Nature, 554, 360-363.

Vellend, M. (2016). The Theory of Ecological Communities. Prinston University Press.

Wootton, J.T. \& Emmerson, M. (2005). Measurement of interaction strength in nature. Annu. Rev. Ecol. Evol. Syst., 36, 419-444.

Ye, H., Clark, A., Deyle, E. \& Munch, S. (2020). rEDM: Applications of Empirical Dynamic Modeling from Time Series.

\section{Supporting information}

Additional Supporting Information may be found in the online version of this article.

\section{Data availability}

Data will be available after the acceptance of this manuscript. 


\section{Author contributions}

K.H., D.H. and T.K. conceived and designed the study. Y.E., Y.S., J.C. and K.H.

collected the data. K.H. analysed the results and prepared figures and tables. K.H., D.H.

and T.K. led writing of the manuscript. K.H., D.H., Y. E, Y.S., J.C., K.G. and T.K.

contributed to revising the earlier draft. All authors have given final approval to submit

this manuscript and agree to be accountable for the aspects of the work that they

conducted.

\section{Competing interests}

The authors declare no competing interests. 
a

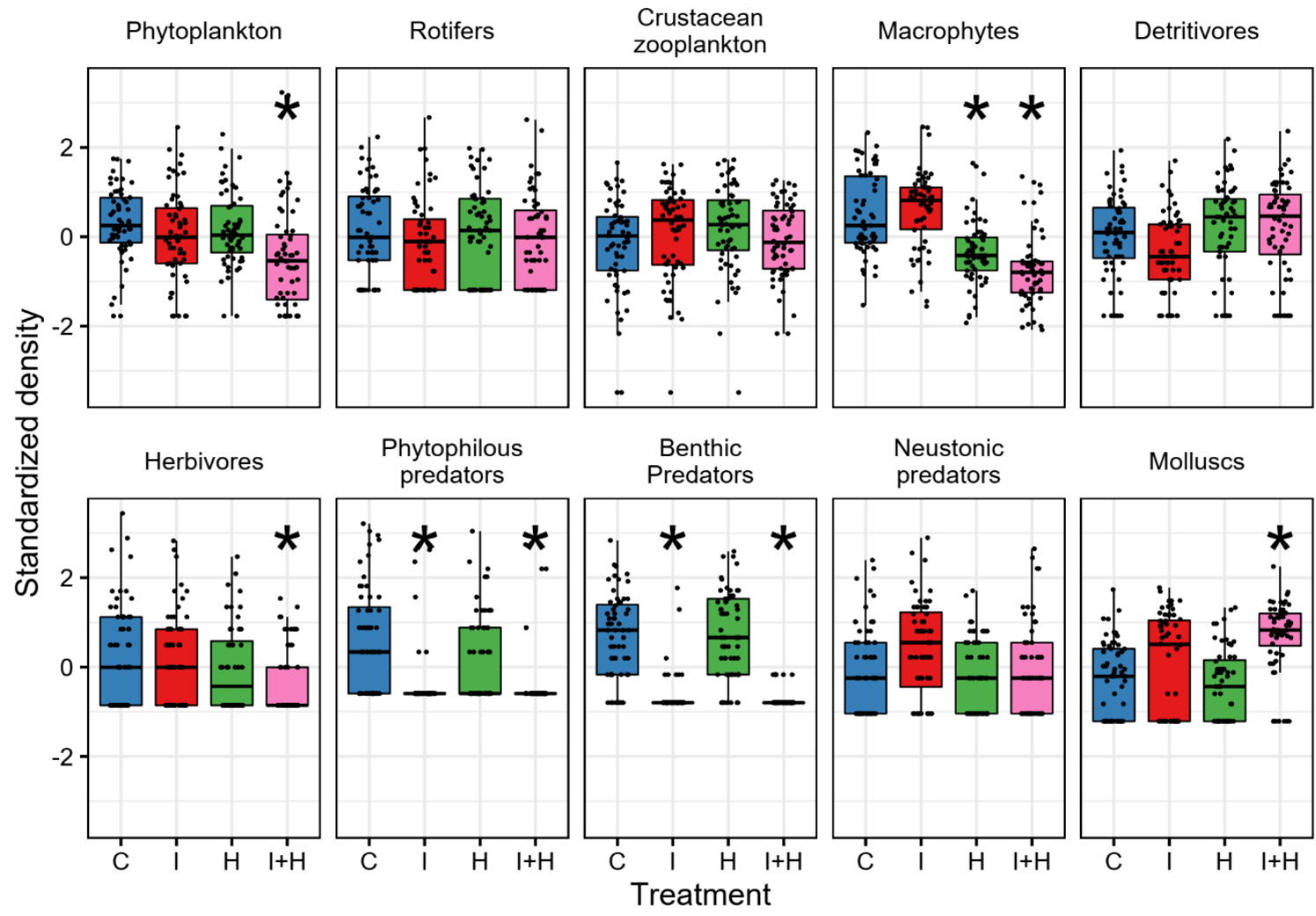

b

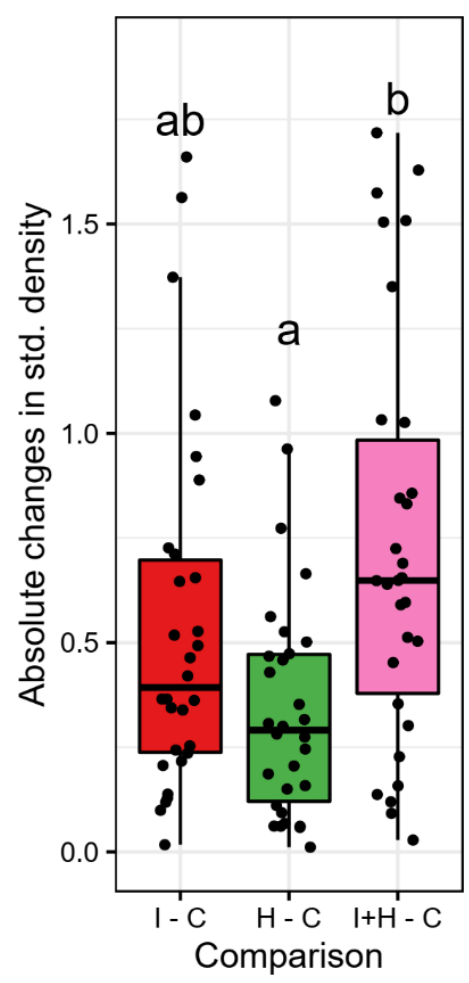

Fig. 1. Pesticide impacts on the density of paddy community members. a, Treatment effects on the density of each paddy community member. Treatment abbreviations are as follows: C: control, I: insecticide alone, $\mathrm{H}$ : herbicide alone, and $\mathrm{I}+\mathrm{H}$ : mixture of insecticide and herbicide. Asterisks indicate significant differences compared to the control $(\alpha=0.05)$. Midline, box limits, and whiskers indicate the median, upper and lower quartiles and $1.5 \times$ interquartile range, respectively. Points indicate the raw values. b, Differences in the treatment impacts on the density of each community member among the three pesticide-applied treatments $(\mathrm{I}, \mathrm{H}, \mathrm{I}+\mathrm{H})$, quantified by absolute changes in the density of each community member due to the pesticide treatments. Changes in the density of each community member were calculated by subtracting the mean density in treatments $\mathrm{I}, \mathrm{H}$, or $\mathrm{I}+\mathrm{H}$ from that of the controls for each year. Different letters indicate significant differences $(\alpha=0.05)$. 
a

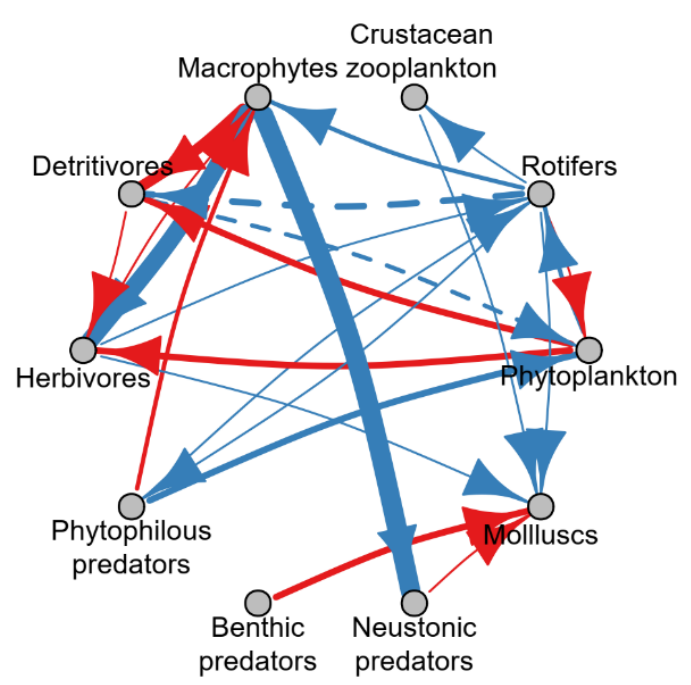

b

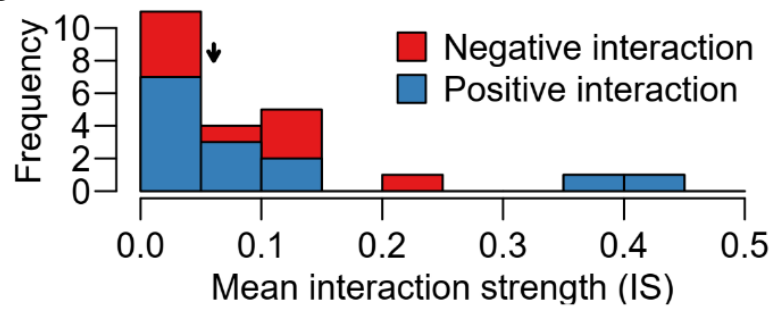

C

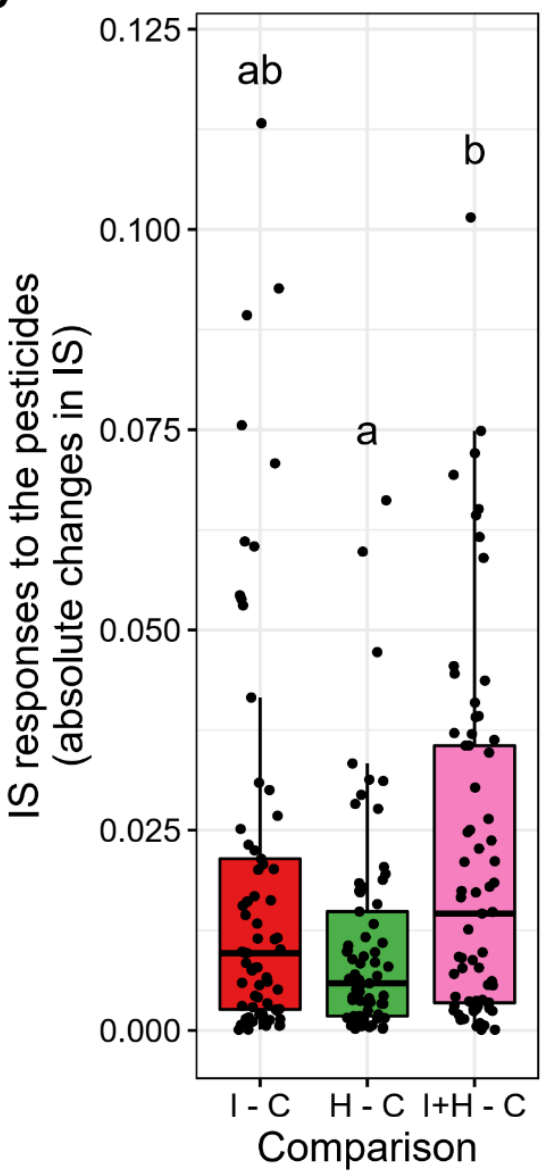

Fig. 2. Reconstructed interaction network of paddy communities and responses in interaction strength (IS) to pesticide treatments. a, Interaction networks of the controls (i.e., those without pesticide disturbances) reconstructed by the EDM analysis. Red and blue arrows indicate negative and positive interactions, respectively. Their thickness is proportional to the IS represented by absolute values of the S-map coefficient averaged over all the experimental periods and replicates. Solid arrows: $P<$ 0.05, dashed arrows: $0.05<P<0.1$. b, Distribution of mean interaction strength averaged over all the experimental periods and replicates in the controls. The vertical arrow indicates the median values. c, Differences in the IS responses to pesticides among the three pesticide-applied treatments. Treatment abbreviations are as follows: $\mathrm{C}$ : 
control, I: insecticide alone, $\mathrm{H}$ : herbicide alone, and $\mathrm{I}+\mathrm{H}$ : mixture of insecticide and herbicide. The IS responses to the pesticides were quantified by absolute changes in the IS due to the pesticide treatments, which were calculated by subtracting the mean strength of each interaction of treatment $\mathrm{I}, \mathrm{H}$, or $\mathrm{I}+\mathrm{H}$ from that of the control for each year. Midline, box limits, and whiskers indicate the median, upper and lower quartiles and $1.5 \times$ interquartile range, respectively. Points indicate the raw values. Different letters indicate significant differences $(\alpha=0.05)$. 


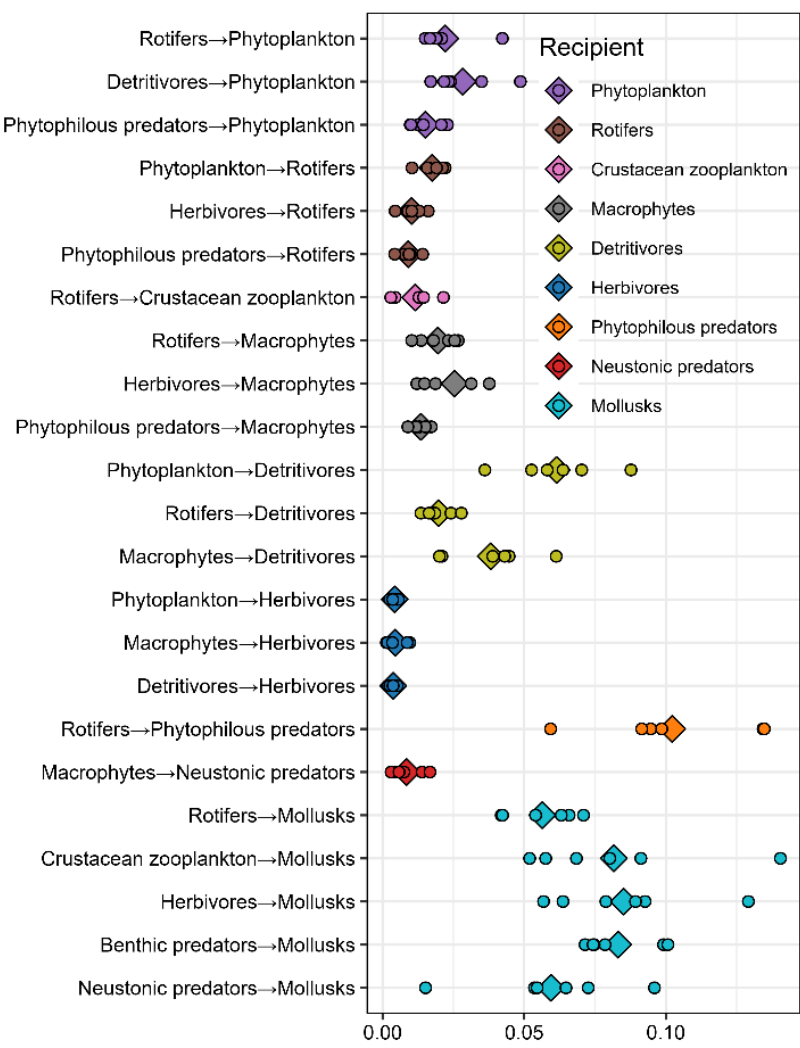

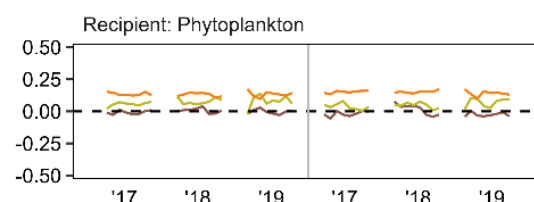
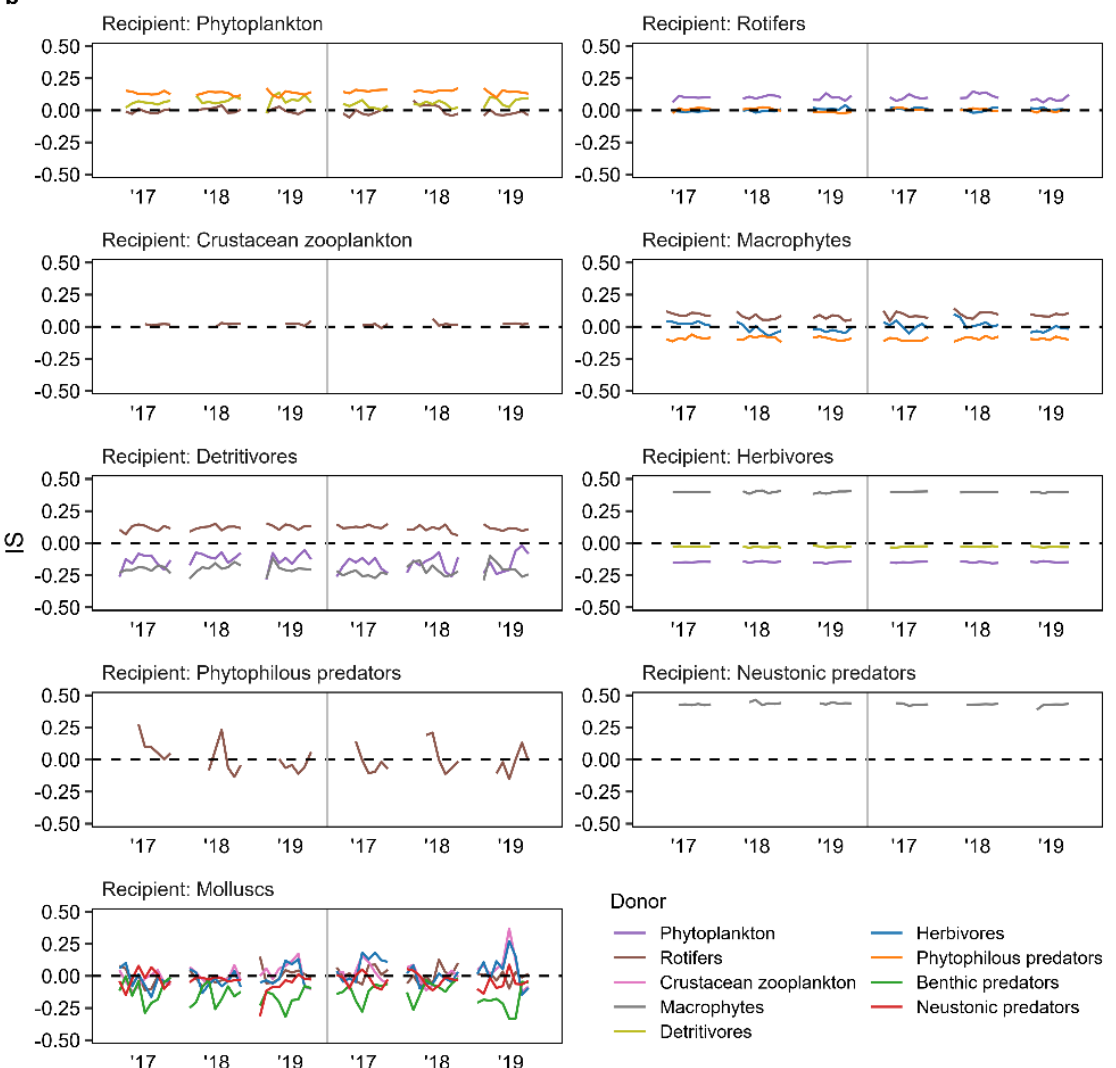

Interaction strength (IS) intrinsic variability (SD in IS of the controls)

Fig. 3. Intrinsic variability in interaction strength (IS) varied by interaction pairs.

IS was quantified by the S-map method. IS intrinsic variability, measured by SD in IS

over the experimental periods in the controls, is an IS characteristic intrinsic to the focal community to which the interaction belongs when disturbances are absent. a,

Comparison of IS intrinsic variability among different interactions. Diamonds and circles indicate the overall means and raw data per replicate per year, respectively. $\mathbf{b}$, Raw timeseries of IS in the controls. Donor and recipient mean initiator and receiver of interaction effects, respectively. The results from different replicates separated by the vertical grey lines are shown. 

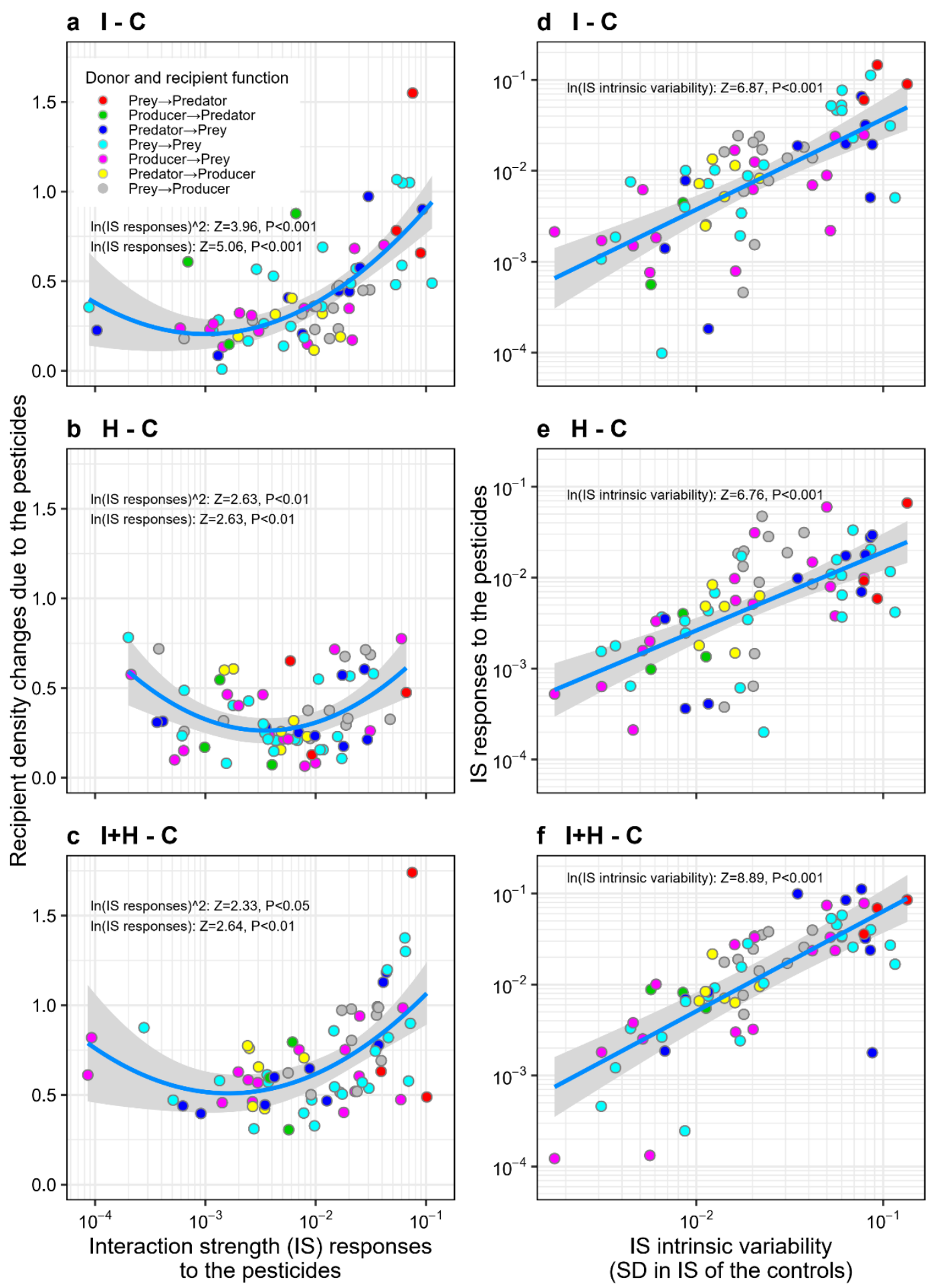

IS intrinsic variability (SD in IS of the controls)

Fig. 4. Density changes in each community member mediated by the two types of variability in interaction strength (IS): IS responses to the pesticides and IS

intrinsic variability. Treatment abbreviations are as follows: C: control, I: insecticide alone, $\mathrm{H}$ : herbicide alone, and $\mathrm{I}+\mathrm{H}$ : mixture of insecticide and herbicide. a-c, Concave 
relationships between IS responses to the pesticides (absolute differences in the strength in each interaction of treatment $\mathrm{I}, \mathrm{H}$, or $\mathrm{I}+\mathrm{H}$ and that in the controls) and changes in interaction recipient density due to the treatments (absolute differences in the density of the recipient of the corresponding interaction pair in treatment $\mathrm{I}, \mathrm{H}$, or $\mathrm{I}+\mathrm{H}$ and that in the controls). d-f, Positive relationships between IS intrinsic variability (SD in IS in the controls) and corresponding IS responses to pesticides. Solid lines and shaded areas indicate conditional model predictions and 95\% confidence intervals, respectively. Each point shows partial residuals. Prediction was done by the most parsimonious models. Figures were generated using the package 'visreg' ver. 2.7.0 (Breheny \& Burchett 2017). 


\section{a I - C}

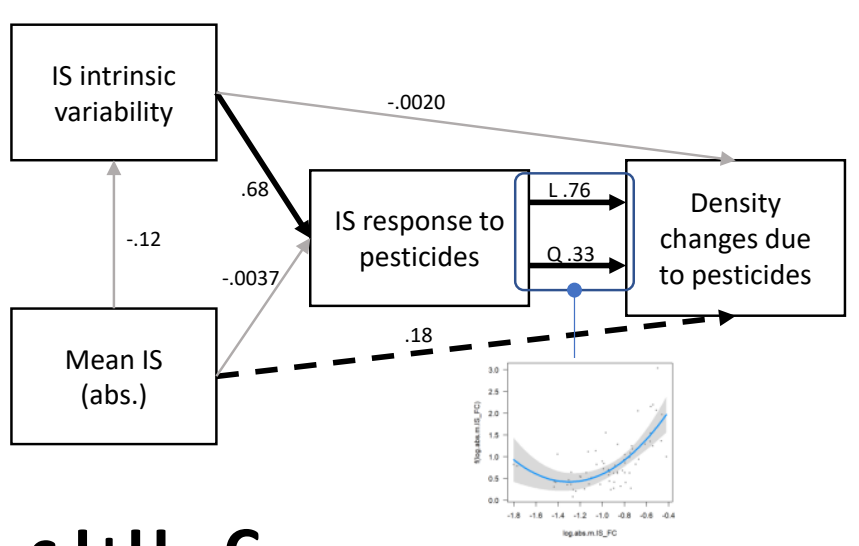

\section{$\mathrm{Cl}+\mathrm{H}-\mathrm{C}$}

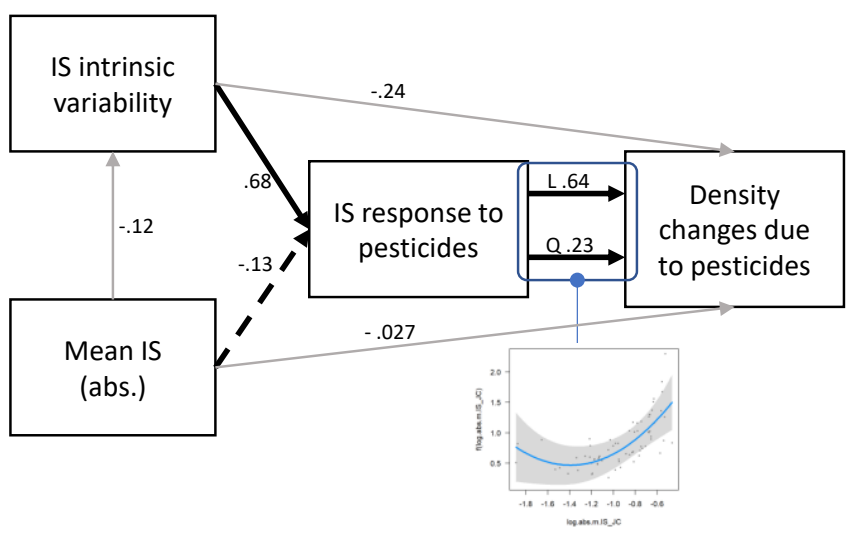

b H - C

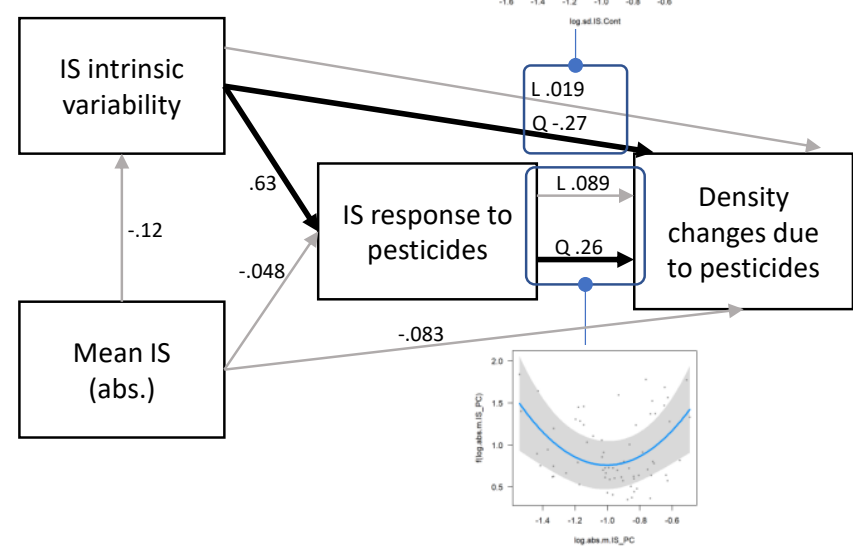

Fig. 5. Cascading linkage from interaction strength (IS) intrinsic variability to density changes via IS responses to pesticides. Hypothetical causal relationships among the parameters of IS and the changes in interaction recipient density due to the pesticide treatments were analysed by structural equation modelling. The effects of a) the insecticide, b) the herbicide and c) the insecticide and herbicide mixture are shown. Treatment abbreviations are as follows: C: control, I: insecticide alone, $\mathrm{H}$ : herbicide alone, and $\mathrm{I}+\mathrm{H}$ : mixture of insecticide and herbicide. L: linear effects, Q: quadratic effects. When causal relationships were nonlinear, actual curves are shown in insets. Solid black, dashed black, and solid grey arrows indicate $P$ values of $<0.05,0.05-0.1$ and $>0.1$, respectively. 